\title{
High-Performance Enhancement-Mode AlGaN/GaN Multi-Channel Power Transistors
}

\author{
Luca Nela ${ }^{1}$, Catherine Erine ${ }^{1}$, Jun $\mathrm{Ma}^{1}$, Halil Kerim Yildirim ${ }^{1}$, Remco Van Erp ${ }^{1}$, Peng Xiang ${ }^{2}$, Kai Cheng ${ }^{2}$, Elison \\ Matioli $^{1}$ \\ ${ }^{1}$ Power and Wide-band-gap Electronics Research Laboratory, EPFL, 1015 Lausanne, Switzerland. \\ ${ }^{2}$ Enkris Semiconductor Inc., Suzhou 215123, China.
}

\begin{abstract}
AIGaN/GaN devices have shown outstanding potential for power conversion applications. However, despite the recent progress, their performance is still far from what the material can offer in terms of on-resistance and breakdown voltage. To address this challenge, here we demonstrate a multi-channel tri-gate High-ElectronMobility Transistor (HEMT) based on an AlGaN/GaN multiple channel heterostructure and a nanostructured gate region. The multi-channel heterostructure leads to a significantly reduced sheet resistance while the nanostructured gate provides excellent control over all the embedded channels and enables to effectively manage the large off-state electric fields. This approach results in e-mode devices with a threshold voltage $\left(V_{\mathrm{TH}}\right)$ of $0.85 \mathrm{~V}$ at $1 \mu \mathrm{A} / \mathrm{mm}$, very low specific on-resistance of 0.46 $\mathrm{mOhm} \cdot \mathrm{cm}^{2}$, and a large breakdown voltage of $1300 \mathrm{~V}$. In addition, we demonstrate multi-channel devices with excellent $V_{\mathrm{TH}}$ stability and reduced current collapse thanks to a novel conformal passivation technique, which shows the potential of the multi-channel tri-gate technology for future power conversion applications.
\end{abstract}

Keywords-GaN HEMTs, AlGaN/GaN, Multi-Channel, TriGate, Surface Passivation, Current Collapse

\section{INTRODUCTION}

A $1 \mathrm{GaN} / \mathrm{GaN}$ devices have shown great potential for efficient power conversion applications thanks to the excellent $\mathrm{GaN}$ material properties. However, despite the recent progress, their performance is still far from the theoretical limits of the material [1]. An effective way to enhance device performance consists of increasing its carrier concentration $\left(N_{\mathrm{s}}\right)$, which directly results in a smaller onresistance $\left(R_{\mathrm{ON}}\right)$. Yet, increasing $N_{\mathrm{s}}$ leads to major challenges for the heterostructure and device design. On the one hand, a larger $N_{\mathrm{s}}$ severely impacts the carrier mobility $(\mu)$, limiting the reduction of the heterostructure sheet resistance $\left(R_{\mathrm{sh}}\right)$. On the other hand, a large carrier concentration results in a difficult control of the channel, which hinders enhancement-

\footnotetext{
This work was supported in part by the Swiss National Science Foundation through Assistant Professor (AP) Energy under Grant PYAPP2_166901, in part by the European Research Council through the European Union's H2020 Program/ERC under Grant 679425, and in part by the ECSEL Joint Undertaking (JU) under Grant 826392. The JU receives support from the European Union's Horizon 2020 research and innovation programme and Austria, Belgium, Germany, Italy, Norway, Slovakia, Spain, Sweden, Switzerland.

L.Nela, C.Erine, J. Ma, H.K. Yildirim, R. van Erp and E. Matioli are with the Power and Wide-band-gap Electronics Research Laboratory (POWERLAB), École Polytechnique Fédérale de Lausanne (EPFL), CH-1015 Lausanne, Switzerland (e-mail: luca.nela@epfl.ch; elison.matioli@epfl.ch).

P.Xiang and K.Chen are with Enkris Semiconductor Inc., Suzhou, China.
}

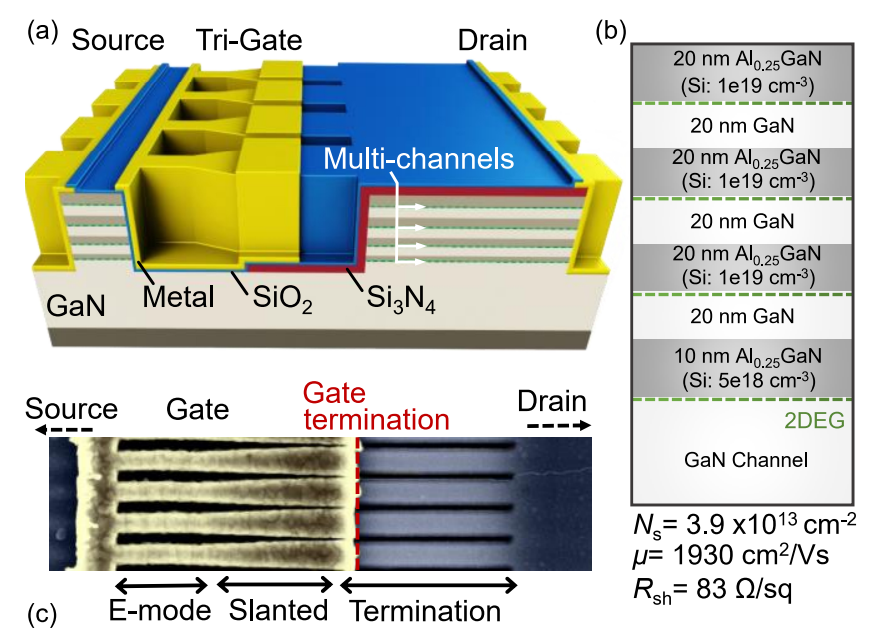

Fig.1. (a) Three dimensional schematics of the multi-channel tri-gate MOSHEMT. (b) Schematics of the multi-channel heterostructure. A $1 \mathrm{~nm}$ thick AlN spacer (not shown) is present between the AlGaN barrier and the $\mathrm{GaN}$ channel. (c) Top SEM view of the nanostructured gate region.

mode (e-mode) operation and degrades the device voltage blocking performance.

A promising approach to address this trade-off is with the use of multi-channel heterostructures [2]-[7], in which several barrier/channel layers are stacked to achieve multiple 2DEGs, which allow to increase the $N_{\mathrm{s}}$ and thus the heterostructure conductivity. While these structures have been proposed for RF applications [8]-[10], their use for power devices depends on very different requirements, which need to be separately addressed. In particular, enhancement-mode (e-mode) operation, large blocking voltage capabilities with reduced leakage current, and good stability during switching operation are fundamental features for power devices, which require careful solutions on a multi-channel platform.

In this work, we address these challenges by demonstrating a high-performance e-mode multi-channel tri-gate HighElectron-Mobility Transistor (HEMT) based on a multiple channel heterostructure and a nanostructured gate region (Fig. 1). The multi-channel heterostructure allows distributing a large $N_{\mathrm{s}}$ in multiple channels, thus maintaining a very high $\mu$ and leading to a much-reduced $R_{\text {sh. }}$. The nanostructured gate [2], [4] provides excellent control over all the embedded channels and enables to effectively manage the large off-state electric field, leading to e-mode devices with very low on-resistance combined with excellent breakdown voltage. In addition, we demonstrate multichannel devices with excellent threshold voltage $\left(V_{\mathrm{TH}}\right)$ stability and reduced current collapse thanks to a conformal surface passivation technique, showing the potential of this technology for future power conversion applications. 

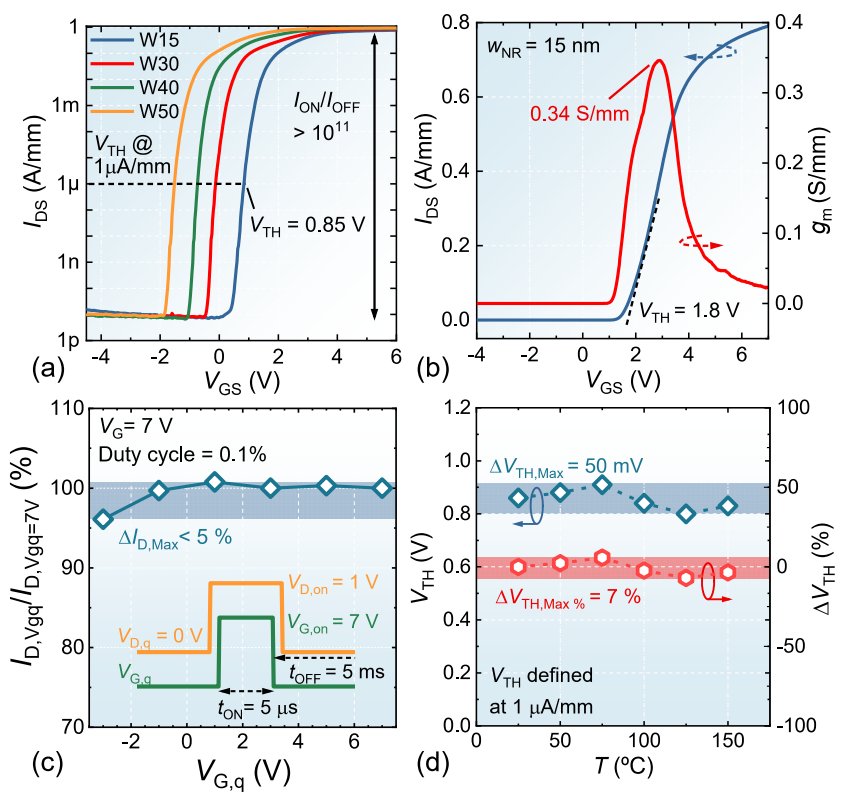

Fig. 2. (a) Transfer curve in logarithmic scale for devices having different nanoribbon width. (b) Linear transfer curve and transcondcutance for a multi-channel device with $w_{\mathrm{NR}}$ of $15 \mathrm{~nm}$. (c) Gate lag measurement (d) $V_{\mathrm{TH}}$ temperature stability for an e-mode multi-channel device.

\section{DEVICE STRUCTURE}

The multi-channel $\mathrm{AlGaN} / \mathrm{GaN}$ heterostructure herein proposed is comprised of 4 parallel 2DEG channels (Fig. 1 (b)). The top 3 channels included $20 \mathrm{~nm}$ AlGaN barrier (Sidoped with a concentration of $\left.10^{19} \mathrm{~cm}^{-3}\right), 1 \mathrm{~nm}$ AlN spacer, and a $20 \mathrm{~nm}$ unintentionally-doped (UID) GaN channel layer, while the last channel featured a $10 \mathrm{~nm} \mathrm{AlGaN} \mathrm{barrier} \mathrm{(Si-}$ doped with a concentration of $5 \times 10^{18} \mathrm{~cm}^{-3}$ ) and $1 \mathrm{~nm}$ AlN spacer. The electron mobility and concentration, extracted by Hall measurement are $1930 \mathrm{~cm}^{2} \cdot \mathrm{V}^{-1} \mathrm{~s}^{-1}$ and $3.9 \times 10^{13} \mathrm{~cm}^{-2}$ respectively, which results in a sheet resistance of $83 \Omega / \mathrm{sq}$. The multi-channel device fabrication started with electronbeam lithography to define the mesa and the nanoribbon in the gate region (Fig. 1 (a-c)), followed by $\mathrm{Cl}_{2}$-based inductively coupled plasma etching (ICP). The etch depth was set to $250 \mathrm{~nm}$. The design of the nanoribbons in the gate region is shown in Fig. 1 (c). Each nanoribbon comprises, from the source to the drain, a $500 \mathrm{~nm}$-long straight portion whose width was tuned to adjust the device threshold voltage $\left(V_{\mathrm{TH}}\right)$ and achieve e-mode operation, a $700 \mathrm{~nm}$-long slanted portion which acts as a field plate and reduces the high-off state electric field, and a $1 \mu \mathrm{m}$-long termination region where the gate electrode is terminated. Reference devices without the slanted portion and with the gate electrode termination on the planar region were fabricated for comparison.

Following the dry etching, a 5-cycle $\mathrm{O}_{2}$ plasma/ $\mathrm{HCl}$ treatment was performed to reduce the etching damages on the nanoribbon sidewalls. A passivation stack comprising 2 $\mathrm{nm}$ of $\mathrm{ALD} \mathrm{SiO} 2$ and $100 \mathrm{~nm}$ of LPCVD $\mathrm{Si}_{3} \mathrm{~N}_{4}$ was deposited [6]. The ALD deposition took place at $300{ }^{\circ} \mathrm{C}$ while the LPVCD deposition occurred at $770{ }^{\circ} \mathrm{C}$ with a flow of $30 \mathrm{sccm}$ of $\mathrm{SiH} 2 \mathrm{Cl} 2$ and $180 \mathrm{sccm}$ of $\mathrm{NH} 3$ at a chamber pressure of $100 \mathrm{mT}$. The $2 \mathrm{~nm}$-thick $\mathrm{SiO}_{2}$ served as an interlayer and protected the $\mathrm{GaN}$ surface from desorption during the LPCVD deposition [11], [12]. The passivation stack was removed in the gate and contact regions with low power RIE etching to enable the formation of tri-gate and tri-ohmic
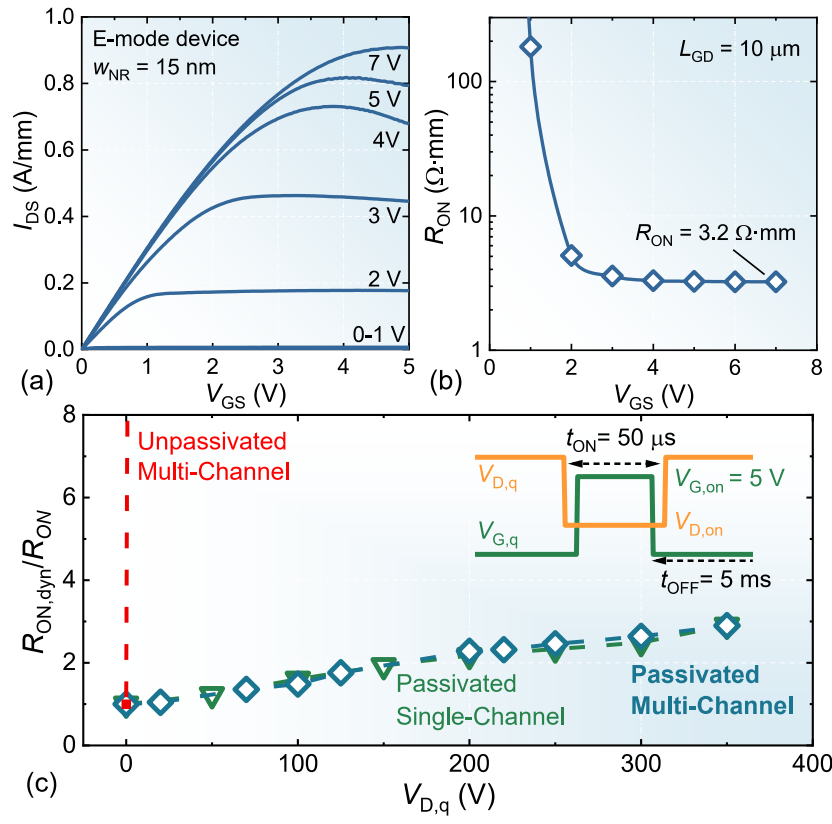

Fig.3. (a) Output curve for a normally-off multi-channel device having $w_{\mathrm{NR}}$ of $15 \mathrm{~nm}$ and $L_{\mathrm{GD}}$ of $10 \mu \mathrm{m}$ (b) On-resistance vs gate voltage extracted from (a). (c) Dynamic on-resistance as a function of the quiescent drain voltage for unpassivated multi-channel devices and passivated multi- and single- channel devices. The top right inset shows the pulse schematics used to perform the measurement.

structures. A Ti/Al/Ti/Ni/Au metal stack was evaporated and annealed at $780{ }^{\circ} \mathrm{C}$ for $30 \mathrm{~s}$ to form the ohmic contacts, followed by the deposition of $25 \mathrm{~nm}$-thick ALD $\mathrm{SiO}_{2}$ layer, which served as the gate oxide. Lastly, a Pt/Au (40 nm / 100 $\mathrm{nm}$ ) gate metal stack was deposited. The device dimensions are $\mathrm{L}_{\mathrm{GS}}=1 \mu \mathrm{m}, \mathrm{L}_{\mathrm{G}}=1.5 \mu \mathrm{m}$ and $\mathrm{L}_{\mathrm{GD}}=10 \mu \mathrm{m}$. Reference single-channel devices were fabricated in the same batch and underwent the same fabrication steps.

\section{DEVICE CHARACTERIZATION}

Figure 2 (a) reports the transfer curve of multi-channel devices with different nanoribbon widths $\left(w_{\mathrm{NR}}\right)$ (see Fig. 1 (c)), which shows that precise control of the device threshold voltage $V_{\mathrm{TH}}$ can be achieved by simply designing the nanoribbon geometry. Besides, the reduction of $w_{\mathrm{NR}}$, in combination with large work-function gate metals [13], enables to reach enhancement-mode operation with $V_{\mathrm{TH}}$ of $0.85 \mathrm{~V}$ at $1 \mu \mathrm{A} / \mathrm{mm}$ for $w_{\mathrm{NR}}$ of $15 \mathrm{~nm}$, despite the large carrier density $\left(3.9 \times 10^{13} \mathrm{~cm}^{-2}\right)$ of the multi-channel heterostructure. The transfer curve of multi-channel devices with $w_{\mathrm{NR}}$ of 15 nm show $V_{\mathrm{TH}}$ of $1.8 \mathrm{~V}$ from linear extrapolation and a large transconductance $\left(g_{\mathrm{m}}\right)$ peak of $0.34 \mathrm{~S} / \mathrm{mm}$ thanks to the large $N_{\text {s. }}$ The threshold voltage stability of the presented multichannel devices was tested both during switching operation and at high temperatures. In particular, a gate lag measurement was performed to characterize the $V_{\mathrm{TH}}$ variation during pulsed conditions. To this end, the device was stressed for a time $t_{\mathrm{off}}=5 \mathrm{~ms}$ at a quiescent gate voltage $V_{\mathrm{G}, \mathrm{q}}$ with a drain bias $V_{\mathrm{D}, \mathrm{q}}$ of $0 \mathrm{~V}$ and then briefly switched-on to $V_{\mathrm{G}, \text { on }}$ of $7 \mathrm{~V}$ and $V_{\mathrm{D} \text {,on }}$ of $1 \mathrm{~V}$ for a time $t_{\mathrm{on}}=5 \mu \mathrm{s}$ during which the drain current $I_{\mathrm{D}}$ was measured (Fig. 2 (c)). A shift of $V_{\mathrm{TH}}$ due to the gate stress would result in a variation of $I_{\mathrm{D}}$. Figure 2 (c) shows a negligible drain current variation of less than $5 \%$ in the whole $V_{\mathrm{G}, \mathrm{q}}$ range between $-2 \mathrm{~V}$ to $7 \mathrm{~V}$ for the multichannel tri-gate devices, which demonstrates their excellent 


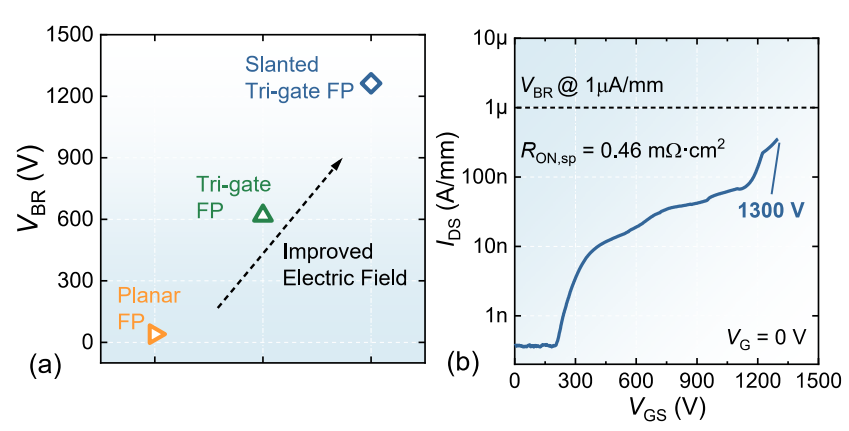

Fig.4. (a) Breakdown voltage for multi-channel devices having planar field plate termination, tri-gate field plate and slanted tri-gate field plate. (b) Offstate leakage current as a function of the drain-to-source voltage $\left(V_{\mathrm{DS}}\right)$, showing breakdown voltage of $1300 \mathrm{~V}$.

$V_{\mathrm{TH}}$ stability during switching operation. The threshold voltage stability was also tested at high temperatures by mounting the device on a heated stage and measuring its transfer curve. A negligible $V_{\mathrm{TH}}$ variation below $50 \mathrm{mV}$ is measured in a temperature range up to $150{ }^{\circ} \mathrm{C}$ (Fig. 2 (d)), which shows good $V_{\mathrm{TH}}$ stability also at elevated temperatures. The output curve for a multi-channel normally-off device with $w_{\mathrm{NR}}$ is reported in Fig. 3 (a) which presents a large saturation current of $900 \mathrm{~mA} / \mathrm{mm}$ and a very low onresistance $\left(R_{\mathrm{ON}}\right)$ of $3.2 \Omega \cdot \mathrm{mm}$, with full device turn-on for gate voltages above $4 \mathrm{~V}$ (Fig. 3 (b)). Such low $R_{\mathrm{ON}}$ for a device with $\mathrm{L}_{\mathrm{GD}}$ of $10 \mu \mathrm{m}$ is possible thanks to the muchreduced sheet resistance of the multi-channel platform compared to conventional single-channel heterostructures. It is of great importance that a low $R_{\mathrm{ON}}$ is maintained also during switching operation. To reduce current collapse, a conformal $\mathrm{SiO}_{2} / \mathrm{Si}_{3} \mathrm{~N}_{4}$ passivation layer was deposited around the nanoribbons terminations (Fig. 1 (a-c)) and in the planar drift region. The dynamic on-resistance $R_{\mathrm{ON} \text {,dyn }}$ was measured with a double-pulse test (DPT) setup. First, the device was stressed in the off-state for a time $t_{\text {off }}=5 \mathrm{~ms}$ with a large drain quiescent voltage $V_{\mathrm{D}, \mathrm{q}}$ and then it was switched on with a $V_{\mathrm{G}}$ of $5 \mathrm{~V}$ for a short time $t_{\text {on }}=50 \mu$ s during which its onresistance was measured (Fig. 3 (c)). While unpassivated multi-channel devices show a large increase of their $R_{\mathrm{ON} \text {,dyn, }}$ even at low $V_{\mathrm{D}, \mathrm{q}}$, such degradation was strongly reduced for passivated multi-channel devices, which presented good dynamic on-resistance up to large $V_{\mathrm{D}, \mathrm{q}}$ of $350 \mathrm{~V}$. Notably, the $R_{\mathrm{ON} \text {,dyn }}$ behavior and values of multi-channel tri-gate devices is similar to the ones of reference single-channel devices with conventional gate electrode termination on the planar drift region, which suggests that further reduction $R_{\mathrm{ON} \text {,dyn }}$ could be achieved by improving the passivation layers quality.

To manage the large off-state field in such highly conducting and three-dimensional heterostructures, novel field plate structures are required. In particular, conventional gate field plates with the gate electrode termination on the planar part of the drift region are not effective due to their inability to deplete all of the embedded channels and result in early device breakdown at about $40 \mathrm{~V}$ (Fig. 4(a)). A more suitable solution for multi-channel structures is achieved by a tri-gate field plate. In this case, the nanoribbon width is enlarged from $\sim 15 \mathrm{~nm}$ (which is required to achieve e-mode operation) to $100 \mathrm{~nm}$ in the termination region (Fig. 1 (c)). The gate electrode is conformally deposited around the nanoribbon with its edge being placed in the termination region, which results in a tri-gate field plate, whose threshold voltage can be set by tuning the nanoribbon width. Thanks to this approach, the breakdown voltage of multi-channel devices can be considerably increased to above $600 \mathrm{~V}$. In addition, by designing a slanted transition between the nanoribbon emode and termination region (Fig. 1 (c)), it is possible to realize a slanted tri-gate field plate [14], [15], which further improves the off-state electric field and resulted in $V_{\mathrm{BR}}$ of $1300 \mathrm{~V}$. In particular, figure 4 (b) shows the off-state leakage for a multi-channel device with slanted tri-gate field plate which presents low current values well below $1 \mu \mathrm{A} / \mathrm{mm}$ and large hard-breakdown at $1300 \mathrm{~V}$. Combined with a much reduced specific on-resistance $\left(R_{\mathrm{ON}, \mathrm{sp}}\right)$ of $0.46 \mathrm{~m} \Omega \cdot \mathrm{cm}^{2}$, this results in a large high-power figure-of-merit of $3.8 \mathrm{GW} / \mathrm{cm}^{2}$, which shows the promising potential of this technology for power conversion applications.

\section{CONCLUSIONS}

In this work, we demonstrated multi-channel tri-gate HEMT based on a multiple channel heterostructure and a nanostructured gate region. Thanks to this approach, e-mode devices showing very low specific on-resistance combined with large breakdown voltage were achieved. Besides, we demonstrated multi-channel devices showing excellent $V_{\mathrm{TH}}$ stability and reduced current collapse. We believe that these results show the promising potential of the multi-channel trigate technology for future power conversion applications.

\section{ACKNOWLEDGMENT}

The authors would like to acknowledge the staff of CMI and ICMP cleanrooms at EPFL for their technical support.

\section{REFERENCES}

[1] B. J. Baliga, "Gallium nitride devices for power electronic applications," Semicond. Sci. Technol., vol. 28, no. 7, p. 074011, 2013, doi: 10.1088/0268-1242/28/7/074011.

[2] J. Ma, C. Erine, P. Xiang, K. Cheng, and E. Matioli, "Multichannel tri-gate normally-on / off AlGaN / GaN MOSHEMTs on Si substrate with high breakdown voltage and low ON-resistance," Appl. Phys. Lett., vol. 242102, pp. 1-5, 2018, doi: 10.1063/1.5064407.

[3] J. Ma, G. Kampitsis, P. Xiang, K. Cheng, and E. Matioli, "MultiChannel Tri-gate GaN Power Schottky Diodes with Low ONResistance," IEEE Electron Device Lett., vol. 40, no. 2, pp. 275278, 2018, doi: 10.1109/LED.2018.2887199.

[4] J. Ma, C. Erine, M. Zhu, L. Nela, P. Xiang, K. Cheng, and E. Matioli, "1200 V Multi-Channel Power Devices with $2.8 \Omega \cdot \mathrm{mm}$ ON-Resistance," 2019 IEEE Int. Electron Devices Meet., 2019, doi: 10.1109/IEDM19573.2019.8993536.

[5] M. Xiao, Y. Ma, K. Cheng, K. Liu, A. Xie, E. Beam, Y. Cao, and Y. Zhang, "3.3 kV Multi-Channel AlGaN/GaN Schottky Barrier Diodes With P-GaN Termination," IEEE Electron Device Lett., vol. 41, no. 8, pp. 1177-1180, 2020, doi: 10.1109/LED.2020.3005934.

[6] L. Nela, H. K. Yildirim, C. Erine, R. Van Erp, P. Xiang, K. Cheng, and E. Matioli, "Conformal passivation of Multi-Channel GaN power transistors for reduced current collapse," IEEE Electron Device Lett., vol. 42, no. 1, pp. 1-1, 2020, doi: 10.1109/led.2020.3038808.

[7] L. Nela, C. Erine, P. Xiang, V. Tileli, T. Wang, K. Cheng, and E. Matioli, "Multi-channel nanowire devices for efficient power conversion," Nat. Electron., 2021, doi: 10.1038/s41928-02100550-8.

[8] R. S. Howell, E. J. Stewart, R. Freitag, J. Parke, B. Nechay, H. Cramer, M. King, S. Gupta, J. Hartman, M. Snook, I. Wathuthanthri, P. Ralston, K. Renaldo, H. G. Henry, and R. C. 
Clarke, "The Super-Lattice Castellated Field Effect Transistor (SLCFET): A novel high performance Transistor topology ideal for RF switching," Int. Electron Devices Meet. IEDM, no. February, pp 11.5.1-11.5.4, 2014, doi: 10.1109/IEDM.2014.7047033.

[9] K. Shinohara, C. King, E. J. Regan, J. Bergman, A. D. Carter, A Arias, M. Urteaga, B. Brar, R. Page, R. Chaudhuri, M. Islam, H. Xing, and D. Jena, "GaN-Based Multi-Channel Transistors with Lateral Gate for Linear and Efficient Millimeter-Wave Power Amplifiers," IEEE MTT-S Int. Microw. Symp. Dig., pp. 1133 1135, 2019, doi: 10.1109/mwsym.2019.8700845.

[10] J. Chang, S. Afroz, K. Nagamatsu, K. Frey, S. Saluru, J. Merkel, S. Taylor, E. Stewart, S. Gupta, and R. Howell, "The super-lattice castellated field-effect transistor: A high-power, high-performance RF amplifier," IEEE Electron Device Lett., vol. 40, no. 7, pp. 1048-1051, 2019, doi: 10.1109/LED.2019.2917285.

[11] M. Hua, S. Member, J. Wei, G. Tang, Z. Zhang, Q. Qian, X. Cai, N. Wang, and K. J. Chen, "Normally-Off LPCVD-SiNx/GaN MIS-FET With Crystalline Oxidation Interlayer," IEEE Electron Device Lett., vol. 38, no. 7, pp. 929-932, 2017, doi: 10.1109/LED.2017.2707473.
[12] M. Hua, Z. Zhang, J. Wei, J. Lei, G. Tang, K. Fu, Y. Cai, B. Zhang, and K. J. Chen, "Integration of LPCVD-SiNxgate dielectric with recessed-gate E-mode GaN MIS-FETs: Toward high performance, high stability and long TDDB lifetime," Tech. Dig. - Int. Electron Devices Meet. IEDM, pp. 10.4.1-10.4.4, 2017, doi: 10.1109/IEDM.2016.7838388.

[13] L. Nela, M. Zhu, J. Ma, and E. Matioli, "High-performance nanowire-based E-mode Power GaN MOSHEMTs with large work- function gate metal," IEEE Electron Device Lett., vol. 40, no. 3, pp. 439-442, 2019, doi: 10.1109/LED.2019.2896359.

[14] J. Ma and E. Matioli, " 2 kV slanted tri-gate GaN-on-Si Schottky barrier diodes with ultra-low leakage current," Appl. Phys. Lett., vol. 112 , no. 5 , 2018, doi: 10.1063/1.5012866.

[15] J. Ma and E. Matioli, "Slanted Tri-Gates for High-Voltage GaN Power Devices," IEEE Electron Device Lett., vol. 38, no. 9, pp. 1305-1308, 2017, doi: 10.1109/LED.2017.2731799. 